\title{
Dynamische Instandhaltungsstrategieanpassung durch Anlagenkritikalitätsbewertung
}

\author{
Theresa Passath und Cornelia Huber
}

Lehrstuhl für Wirtschafts- und Betriebswissenschaften, Montanuniversität Leoben, Leoben, Österreich

Eingegangen 6. November 2018; angenommen 11. Dezember 2018; online publiziert 9. Januar 2019

Zusammenfassung: Durch die immer steigende Systemkomplexität und zunehmende Automatisierung wird dem Asset an sich, vor allem bedingt durch die einhergehende Substitution des Produktionsfaktors Arbeit (Mensch) durch den Produktionsfaktor Anlage (Maschine), immer mehr Bedeutung zugesprochen. Um die wertschöpfungsorientierte Gestaltung des Instandhaltungsmanagements im Sinne des Lean Smart Maintenance Gedankens weiterhin verfolgen zu können, ist es wichtig, auf Grund der sich ständig ändernden Umfeldbedingungen sowie der damit verbundenen Veränderung der Instandhaltungsziele, die Instandhaltungsstrategie dynamisch an diese Veränderungen anzupassen, um eine ressourceneffiziente Anlagenbewirtschaftung zu ermöglichen. In diesem Zusammenhang kommt der dynamischen Anlagenkritikalitätsbewertung eine hohe Bedeutung zu. Auf Basis der Bewertungsergebnisse kann die Instandhaltungsstrategie dynamisch angepasst sowie u. a. eine anlagenspezifische Budgetplanung durchgeführt werden.

Schlüsselwörter: Kritikalitätsbewertung, Dynamische Instandhaltungsstrategieanpassung, Asset Management System, Ressourceneffiziente Anlagenbewirtschaftung

Dynamic Maintenance Strategy Adjustment through Asset Criticality Assessment

Abstract: Due to the increasing system complexity and rising automation, the asset itself is gaining more importance due to the accompanying substitution of the production factor labour (human) by the production factor asset (machine). In order to be able to continue to pursue this value-adding design of maintenance management in the sense of the Lean Smart Maintenance concept, it is important to dynamically adapt the maintenance strategy to

DI T. Passath ( $\square)$

Lehrstuhl für Wirtschafts- und Betriebswissenschaften,

Montanuniversität Leoben,

Peter-Tunnerstraße 25-27,

8700 Leoben, Österreich

theresa.passath@unileoben.ac.at these changes and to enable resource-efficient asset management because of the constantly changing environmental conditions and the associated changes in maintenance targets. As an assistance for a dynamic adaption of the maintenance strategy, the criticality analysis as well as the subsequent risk analysis of the asset's components can be used. Based on the results of the criticality analysis, the maintenance strategy can be dynamically adapted as well as asset-specific budget decisions can be made.

Keywords: Criticality analysis, Dynamic maintenance strategy adjustment, Asset Management System, Resource-efficient asset-management

\section{Einleitung}

Bedingt durch die fortschreitende Digitalisierung wird dem Asset mehr Wertigkeit zugesprochen sowie strategische Entscheidungen und Stakeholder Anforderungen bei Strategieentscheidungen werden miteinbezogen [1-3]. Physische Anlagen sollen nicht mehr nur Teil des Betriebs sein, sondern als eigenständige Wertschöpfungsressourcen wahrgenommen werden, was auch ein Charakteristikum eines modernen Instandhaltungsmanagements darstellt $[1,4,5]$. Dem kann die Einführung eines strategischen Asset Management Systems Hilfe leisten. Bei einem strategischen Asset Management System wird der Fokus auf die Lebenszyklusbetrachtung, beginnend bei der Investitionsbis hin zur Aussonderungsphase der Anlage, gelegt mit dem Ziel, die Lebenszykluskosten dauerhaft zu minimieren und Verfügbarkeiten zu maximieren [1,6]. Dieser Ansatz wird durch die ISO 55000 Norm und die DIN EN 16646 bekräftigt, welche ein Asset Management System über alle drei Managementebenen - normativ, strategisch und operativ - voraussetzt, um die strategischen Erfolgsfaktoren Kosten, Qualität, Zeit, Flexibilität, Agilität, Sicherheit und Umweltschutz positiv zu beeinflussen und die Wettbewerbsvorteile des Unternehmens zu sichern [1, 6-8].

Um die Ziele eines strategischen Asset Managementsystems zu erreichen, sind die Wahl der geeigneten Instandhal- 
Abb. 1: Regelkreis der Instandhaltung [8]

Abb. 2: Ergebnisse Benchmarkstudie 2018 zur anlagenbezogenen Strategieplanung [2]

Abb. 3: Daten und Informationen im Zeitalter von Industrie $4.0[9,10]$
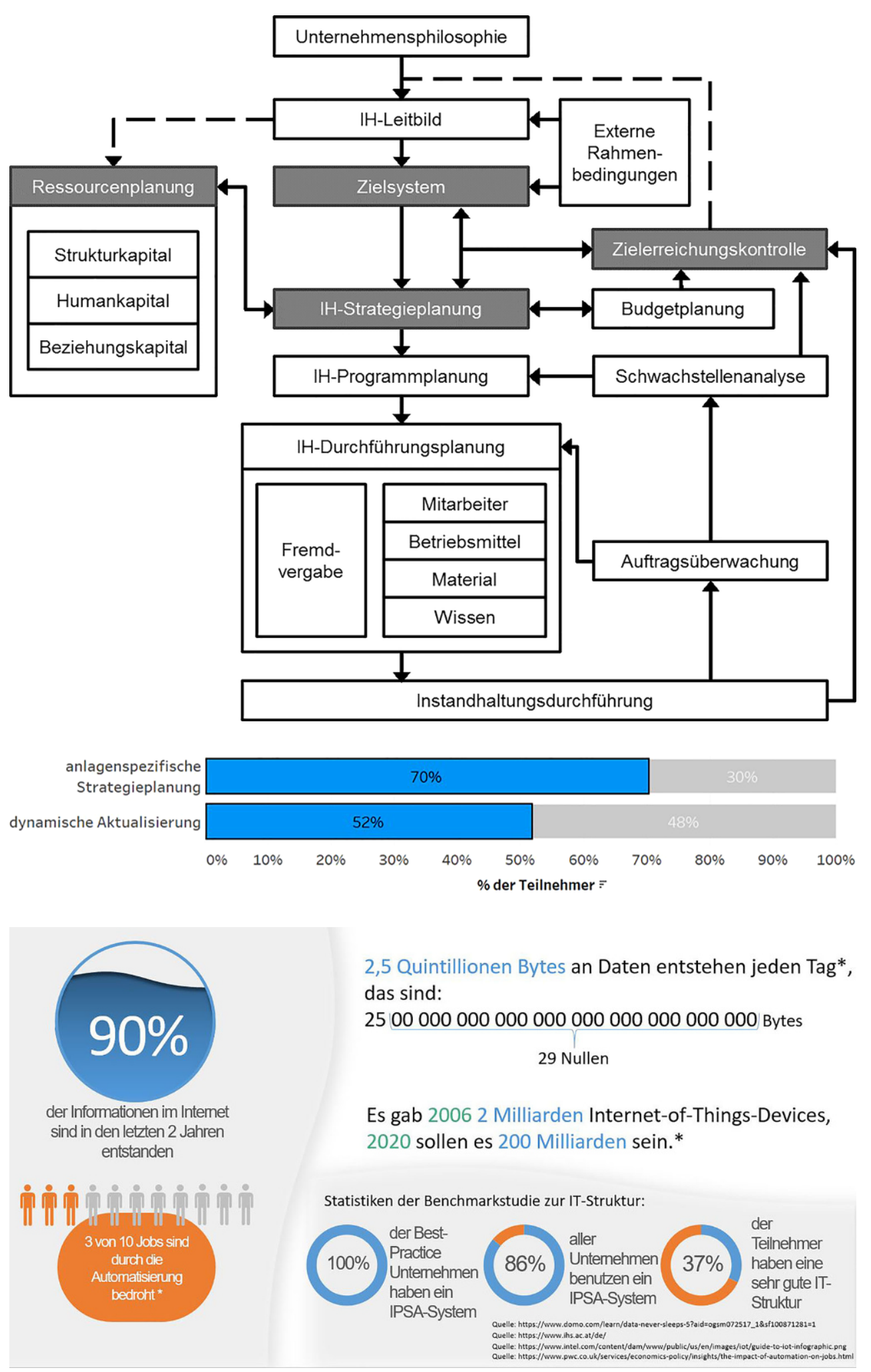
Abb. 4: Prozessmodell der dynamischen Instandhaltungsstrategieoptimierung [5]

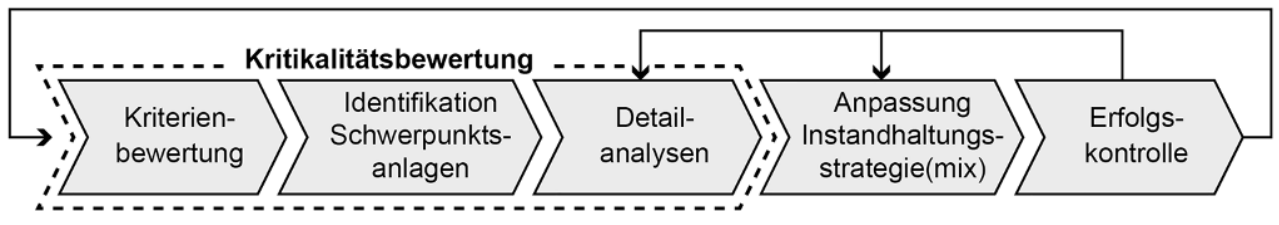

gezielte Maßnahmenableitung eine Reduktion der Kritikalität bzw. der Kosten zu erreichen sowie eine hohe Verfügbarkeit dauerhaft sicherzustellen. tualisierung in Richtung Wertschöpfungsorientierung, welche die zentrale Rolle im Regelkreis der Instandhaltung bildet, unerlässlich (Abb. 1; $[5,8]$ ).

Umso wichtiger ist es für produzierende Unternehmen, die sich bietenden Möglichkeiten der Digitalisierung durch vertikale und horizontale Integration der Daten- und Informationssysteme zu nutzen [6]. Dennoch ist der aktuelle Stand der Industrie ein anderer, wie die Ergebnisse einer aktuellen Benchmarkstudie zeigen (siehe Abb. 2).

Von den 134 befragten Unternehmen passen bereits $70 \%$ die Instandhaltungsstrategie der unternehmensspezifischen Situation an und betreiben somit eine anlagenspezifische Strategieplanung. Jedoch verzichten $30 \%$ noch auf diese Maßnahme. Zusätzliches Entwicklungspotenzial besteht auch in der strategischen Ausrichtung, da nur jedes zweite Unternehmen den dynamischen Ansatz der Strategieplanung verfolgt [2]. Für einen dynamischen Ansatz sind IT gestützte Systeme empfehlenswert, um den Anforderungen nach Analysetools für das Handling großer Datenmengen, gerecht zu werden. Daher muss auch der Transfer relevanter Daten an Bedeutung zunehmen. Dies wird durch die Ergebnisse einer OECD-Studie untermauert, in der verschiedene Aspekte der Digitalisierung zusammengefasst wurden, wie in Abb. 3 ersichtlich $[9,10]$.

Datenmengen steigen exponentiell, sodass es nahezu unmöglich wird, Daten in annehmbarer Zeit manuell zu analysieren, verifizieren und auszuwerten. Zusätzlich müssen Unternehmen eine hohe Flexibilität aufweisen, um den sich stetig ändernden Umweltbedingungen und Kundenanforderungen gerecht zu werden. Dieser Fortschritt in Richtung Digitalisierung wird zukünftig auch für österreichische Betriebe ein entscheidender Wettbewerbsfaktor sein [11]. Hierfür bietet sich eine dynamische Modellierung und Auswertung verfügbarer Daten an, wie es auch eine dynamische Kritikalitätsbewertung als ersten Schritt der Optimierung des anlagenspezifischen Instandhaltungsstrategiemix fordert (Abb. 4).

Ziel solch einer Kritikalitätsbewertung durch automatische Datenaufbereitung in Kombination mit Expertenwissen sollte sein, kritische Anlagen zu identifizieren, um durch

\section{Kritikalitätsbewertung}

Studien belegen, dass die Instandhaltungsstrategie aktuell meist auf Herstellerinformationen bzw. auf Basis von langjährigen Erfahrungswerten (89\%) oder historischen Daten (66\%) angepasst wird. Selten wird eine Anlagen- (34\%) und Risikobewertung (53\%) zur Strategiewahl herangezogen, wie die Benchmarkstudie verdeutlicht (Abb. 5; [2]).

Somit ist eine strukturierte Vorgehensweise meist selten vorhanden, was aber dem Grundgedanken eines Asset Management Systems bzw. der Lebenszyklusbetrachtung widerspricht. Umso wichtiger ist es, bedingt durch die stetig steigenden Datenmengen, eine objektive, wenn möglich eine dynamische Anlagenbewertung zu betreiben, um sich einerseits rechtzeitig an die sich ändernden Umfeldbedingungen (Veränderungen Absatzmarkt) anzupassen, aber andererseits auch um alle kritischen Anlagen echtne dynamische Anlagenbewertung sind neben einer guten und einheitlichen Datenqualität auch eine Datenerfassung auf Anlagen- bzw. Komponentenebene. Des Weiteren ist eine einheitliche Anlagenstruktur für die Datenauswertung unerlässlich. Durch eine direkte Schnittstellenanbindung zu einem ERP-System, wie z. B. SAP, kann für die Auswertung auf Echtzeitdaten zurückgegriffen werden [1, 8, 12].

Solch eine kritikalitätsbezogene Anlagenbewertung besteht im Wesentlichen aus drei Schritten. Im ersten Schritt, der Kriterienbewertung, werden die Anlagen an Hand von quantitativen Kriterien auf Basis von Echtzeitdaten eines ERP Systems, angelehnt an die Erfolgsfaktoren des Unternehmens, bewertet. Anschließend wird der Anlagenindex je Anlage ermittelt, der die Kritikalität der Anlage quantifiziert und das Potenzial indirekter Instandhaltungskosten (Ausfallskostenpotenzial) repräsentiert [8, 13]. Je höher der Anlagenindex [14], desto kritischer ist die bewertete Anlage einzustufen $[6,8]$. zeitbasiert zu identifizieren. Voraussetzungen für solch ei-
Abb. 5: Ergebnisse der Benchmarkstudie 2018 zur Wahl der Instandhaltungsstrategie [2]

\section{Erfahrung fließt am stärksten in die Instandhaltungsstrategiewahl ein}

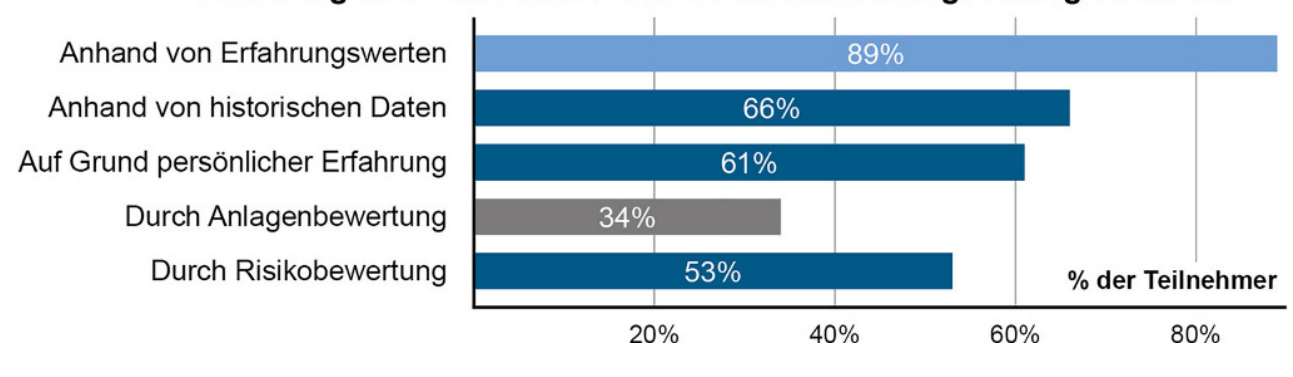




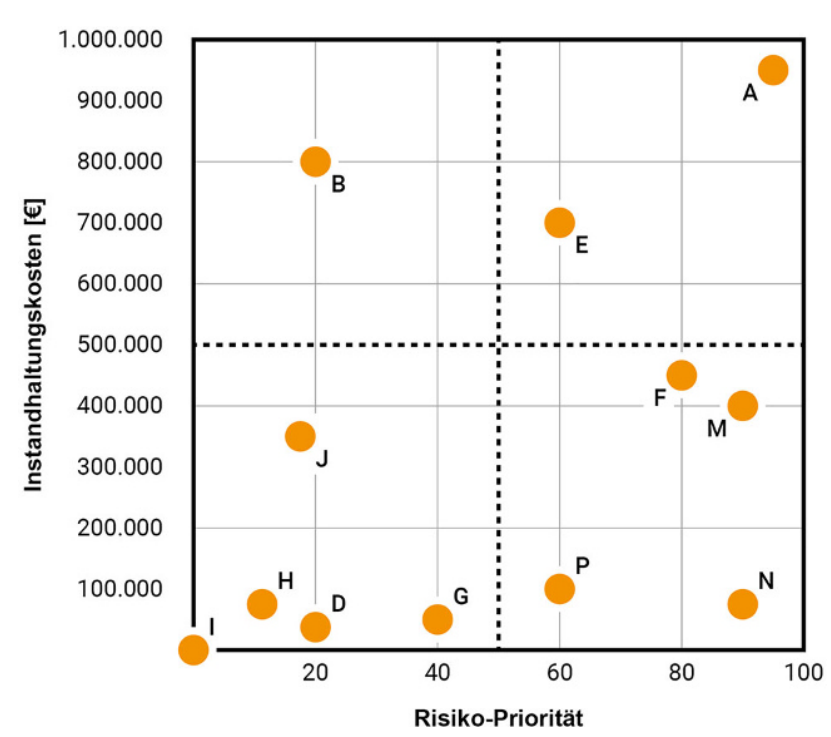

Abb. 6: Anlagenprioritätsportfolio [15]

Um die kritischsten Anlagen, die das größte Potenzial zur Erhöhung des Wertschöpfungsbeitrags haben, zu identifizieren, wird im zweiten Schritt ein Portfolio generiert, in dem der Anlagenindex den direkten Instandhaltungskosten gegenüber gestellt wird. Das Anlagenprioritätsportfolio liefert somit einen genauen Überblick über die kosten- sowie risikokritischen Anlagen (Abb. 6).

Die Detailanalyse, die den dritten Schritt der Kritikalitätsbewertung bildet, werden für zuvor als kosten- und risikoreich identifizierten Anlagen durchgeführt.

\section{Detailanalysen der Schwerpunktanlagen}

Ziel der Detailanalysen ist es, alle kosten- und risikobehafteten Anlagen durch gezielte Anpassung der Instandhaltungs-Strategie in den linken unteren Quadranten zu verschieben. Anlagen oberhalb der unternehmensspezifisch definierten Kostengrenze werden als kostenkritisch angese- hen. Hier gilt es, kostensenkende Maßnahmen abzuleiten. Hingegen sollten bei Anlagen rechts der vertikalen Trennlinie Maßnahmen zur Risikoreduktion und Senkung des Anlagenindex definiert werden $[5,8,20]$.

Bei kostenkritischen Anlagen werden deren Instandhaltungskosten in die Einzelkostenarten (Material-, Personal-, Fremdkosten) zerlegt, die größten Kostentreiber identifiziert und Maßnahmen zur Kostenreduktion abgeleitet.

Bei den risikoreichen Anlagen ist es sinnvoll, die Kriterienbewertung eine Ebene tiefer auf Komponentenebene zu wiederholen, um auch die kritischen Komponenten zu identifizieren und den Risikobereich somit einzugrenzen. In weiterer Folge wird für die risikoreichsten Komponenten eine kausalitätsbezogene Schwachstellenanalyse angewandt. Hierbei werden mittels RMEA (Risk Mode and Effect Analysis) aktuelle sowie potenzielle Risiken identifiziert und nach dem Schadensausmaß, der Auftretenshäufigkeit und der Entdeckungswahrscheinlichkeit bewertet. Als Ergebnis dieser Bewertung erhält man eine Risikoprioritätszahl (RPZ), die je nach Höhe den Risikograd darstellt, für welchen infolgedessen Maßnahmen zur Risikoreduktion definiert werden müssen. Gleich wie für die Kritikalität gilt in diesem Fall auch, je höher die RPZ, desto gravierender ist das Risiko $[5,15]$.

\section{Dynamische Instandhaltungsstrategiean- passung und Budgetierung}

Das Ergebnis der Detailanalysen (Risiko- und Kostenanalyse) bildet die Grundlage für die Anpassung der Instandhaltungsstrategie (Abb. 7). In erster Linie fokussiert man sich auf Anlagen mit einem hohen Risiko- und auch hohen Kostenpotenzial, die kritischsten Anlagen und solche, bei denen die Detailanalysen einen Verdacht einer falschen Instandhaltungsstrategie bestätigt haben. Die detaillierte Kostenanalyse liefert Informationen darüber, ob die angefallenen Kosten Berechtigung haben (z. B. große Reparatur) und somit keine kostenreduzierenden Maßnahmen gesetzt werden müssen oder ob die analysierte Anlage unbe-

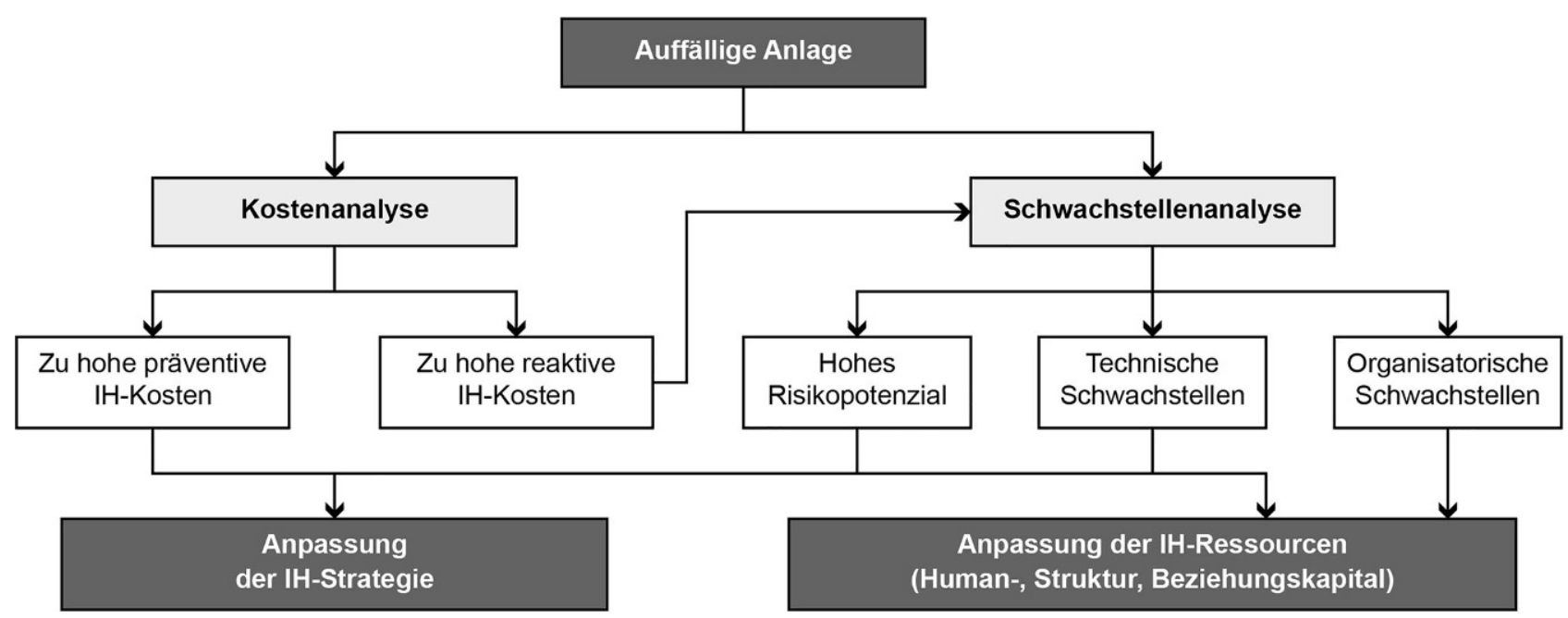

Abb. 7: Dynamische Instandhaltungsstrategieanpassung auf Basis der Detailanalysen [4] 
gründete hohe präventive und/oder reaktive Kosten besitzt sowie welche Tätigkeiten dafür verantwortlich waren. Bei hohen reaktiven Kosten müssen die entsprechenden Tätigkeiten genauer analysiert werden, wobei bei enormen präventiven Kosten der größte Kostentreiber identifiziert werden muss. Dementsprechend können organisatorische Maßnahmen (Anpassung der Wartungs- und Inspektionsintervalle, Auslagerung von Tätigkeiten, und mehr) gesetzt werden. Die Alternative mit dem günstigsten Kosten-Risiko-Verhältnis ist als neue Strategie zu wählen $[5,8,20]$.

Auf Basis des Ergebnisses der Risikobewertung, der RPZ je Fehler, werden im nächsten Schritt im Team Maßnahmen definiert, dieses Risiko durch Minderung des Schadensausmaßes, der Auftretenshäufigkeit aber auch der Entdeckungswahrscheinlichkeit zu minimieren. Des Weitern gilt es, für die abgeleiteten Maßnahmen die zusätzlich angefallenen Instandhaltungskosten, aber auch das Kosteneinsparungspotenzial bedingt durch die Fehlerbehebung abzuschätzen. Maßnahmen mit einem positiven Einsparungspotenzial sind umzusetzen, beginnend bei der effektivsten bzw. schnellst umsetzbarsten $[5,8,15]$.

Um den Erfolg der dynamischen Instandhaltungsstrategieanpassung garantieren zu können, muss das Budget dementsprechend dynamisch hinsichtlich Integration von Maßnahmen zur Risiko- und Kosteneinsparung anpassbar sein, was in den meisten Betrieben noch nicht der Fall ist. Um solche Anforderungen einer dynamischen und wertschöpfungsorientieren Instandhaltung nachzukommen, ist es unerlässlich, die Ergebnisse der Risikobewertungen, welche im Zuge der Instandhaltungsstrategieplanung und -anpassung stattfinden, als Input für Budgetentscheidungen heranzuziehen. Da abgeleitete Instandhaltungsmaßnahmen nach ihrem Bewertungsergebnis priorisiert und geplant werden, können priore Maßnahmen durch die dynamische Budgetierung in die aktuelle Planungsperiode übernommen werden und weniger priore bzw. bereits geplante in die nächste Periode verschoben werden. Somit kann trotz dynamischer Budgetplanung das Instandhaltungsbudget eingehalten werden $[1,16]$.

\section{Zusammenfassung und Ausblick}

Eine dynamische Kritikalitätsbewertung bietet eine Grundlage für die Unterstützung bei anlagenwirtschaftlichen Entscheidungen, für eine gezielte Schwachstellenanalyse und eine Optimierung der Instandhaltungsstrategie. Durch die automatisierte, auf Echtzeitdaten basierende Bewertungsmethodik wird auch der damit verbundene Bewertungsaufwand auf ein Minimum reduziert. Zusätzlich wird ein Mehrwert und Nutzen durch das Aufzeigen aktueller sowie potenzieller Risiken generiert [17, 18]. Dadurch sind eine Erhöhung der Planungssicherheit und eine Steigerung der Effizienz gegeben. Anlagen können somit einfach und effektiv im Hinblick auf Risiko und Kosten klassifiziert werden. Eine einheitliche Anlagenbewertung dient als Entscheidungsgrundlage für Budgetplanungen, Investitionsentscheidungen und für die Optimierung der Anlage über den gesamten Lebenszyklus hinweg, demzufolge kann das Ziel einer ressourceneffizienten Anlagenbewirtschaftung, die bestmög- lichste Sicherheit und Wirtschaftlichkeit zu gewährleisten, erreicht werden [19].

Funding. Open access funding provided by Montanuniversität Leoben.

Open Access Dieser Artikel wird unter der Creative Commons Namensnennung 4.0 International Lizenz (http://creativecommons.org/licenses/ by/4.0/deed.de) veröffentlicht, welche die Nutzung, Vervielfältigung,

Bearbeitung, Verbreitung und Wiedergabe in jeglichem Medium und Format erlaubt, sofern Sie den/die ursprünglichen Autor(en) und die Quelle ordnungsgemäß nennen, einen Link zur Creative Commons Lizenz beifügen und angeben, ob Änderungen vorgenommen wurden.

\section{Literatur}

1. Passath, T.; Kinz, A.: Einführung eines strategischen Asset Management Systems am Beispiel der Zellstoffindustrie, in Biedermann, H.(Hrsg.): Predictive Maintenance - Realität und Vision, Köln: TÜV Media GmbH, 2018, S. 41-64

2. Kinz, A.: Bewertungskategorie "Instandhaltungsstrategie“" in Biedermann, H. (Hrsg.): Benchmark - Instandhaltung, Eine Studie zum Reifegrad von Instandhaltungsorganisation der DACH Region, TÜV Media GmbH, Köln, 2018, S. 68-76

3. Minnaar, J. R.; Basson, W.; Vlok, P.-J.: Quantitative methods required for implementing PAS55 or the ISO 55000 series for asset management, The South African Journal of Industrial Engineering, 24 2013, Nr. 3. https://doi.org/10.7166/24-3-576

4. Picot, A; Maier, M.: Analyse- und Gestaltungskonzepte für das Outsourcing, Die Fachzeitschrift für Informationsmanagement o. Jg., 1992, Nr. 4, S. 14-27

5. Kinz, A.; Schröder W.; Passath, T.; Praher, P.; Freudenthaler, B.: Dynamisierung und wertschöpfungsorientierte Optimierung der Instandhaltungsstrategie, Instandhaltungs-Berater, 74. Akt., Köln: TÜV Media Gmbh, 2017, S.1-51

6. Kinz, A.; Passath T.: Ressourceneffiziente Anlagenbewirtschaftung, WINGbusiness, 2018, Nr. 1/18, S. 29-32

7. Biedermann, H.: Predictive Maintenance - Möglichkeiten und Grenzen, in Biedermann, H. (Hrsg.): Predictive Maintenance - Realität und Vision, Köln: TÜV Media GmbH, 2018, S. 23-40

8. Kinz, A.: Ausgestaltung einer dynamischen, lern- und wertschöpfungsorientierten Instandhaltung, Diss., Leoben, Montanuniv. Lehrst. f. Wirtschafts- und Betriebswissenschaften, 2017

9. OECD: OECD Economic Surveys: Austria 2017, OECD Publishing, Paris 2017

10. Kühnast, R.: Bewertungskategorie "IT-Struktur", in Biedermann, H. (Hrsg.): Benchmark - Instandhaltung, Eine Studie zum Reifegrad von Instandhaltungsorganisation der DACH Region, Köln: TÜV Media $\mathrm{GmbH}, 2018$, S. 77-86

11. Biedermann, $H$.: Smart maintenance: intelligente, lernorientierte Instandhaltung; Biedermann H. (Hrsg.): Praxiswissen für Ingenieure Instandhaltung, 29. Instandhaltungsforum, Köln: TÜV Media Verlag, 2015

12. Bernerstätter, R.; Nemeth, T.; Glawar, R.; Habersohn, C.; Biedermann, H.: Instandhaltung 4.0 - Sicherung der Produktqualität und Anlagenverfügbarkeit durch einen echtzeitbasierten Instandhaltungsleitstand, WINGbusiness, 2016, Nr. 1/2016, S. 25-28

13. Kinz, A., Biedermann, H.; Anlagenspezifische Instandhaltungsstrategiewahl durch strukturierte Anlagenbewertung, in Biedermann, H.(Hrsg.): Smart Maintenance: intelligente, lernorientierte Instandhaltung, Köln: TÜV Media GmbH, 2015, S. 221-238

14. Schröder, W.: Ganzheitliches Instandhaltungsmanagement: Aufbau, Ausgestaltung, Bewertung, 1. Aufl., Wiesbaden, 2010

15. Kinz, A.; Bernerstätter, R.: Instandhaltungsoptimierung mittels Lean Smart Maintenance: Einführung des Lean Smart Maintenance Ansatzes, in Biedermann, H. (Hrsg.): Lean Smart Maintenance: Konzepte, Instrumente und Anwendungen, Köln: TÜV Media GmbH, 2016, S. $61-100$

16. Biedermann, H.; Esfandeyari, O.: Instandhaltungsbudgetierung Verfahren und Methoden zur Planung von Budgets der Anlageninstandhaltung, Noch nicht veröffentlicht 
17. Balzer, G.; Schorn, C.: Asset Management für Infrastrukturanlagen: Energie und Wasser. 2. Aufl, Berlin: Springer Vieweg, 2014

18. Biedermann, H.; Kinz, A.; Bernerstätter, R.; Zeller, T.: Lean Smart Maintenance - Umsetzung einer schlanken, lernorientierten, risikound ressourcenoptimierten Instandhaltung in der Prozessindustrie, Productivity, 21, 2016, Nr. 2, S. S. 41-43

19. Schröder, W.; Kleindienst, B.: Resource Risk Based Maintenance Ein Vorgehensmodell und dessen beispielhafte Umsetzung in der Stahlindustrie, in Biedermann, H. (Hrsg.): Ressourceneffizientes An- lagenmanagement: der Beitrag von Instandhaltung und Anlagenwirtschaft zu einem effizienten Umgang mit Ressourcen, Köln: TÜV Media Verlag, 2013, S.77-103

20. Kinz, A.; Schröder, W.; Passath, T:; Praher, P.; Freudenthaler, B. Optimierung des anlagenspezifischen Instandhaltungsstrategiemix am Beispiel eines komplexen Fertigungssystems, in Biedermann, H. (Hrsg.): Erfolg durch Lean Smart Maintenance - Bausteine und Wege des Wandels, Köln: TÜV Media GmbH, 2017, S.185-216 